\title{
GROUPS WITH COMPLETELY REDUCIBLE REGULAR REPRESENTATION
}

\author{
LARRY BAGGETT $^{1}$ AND KEITH TAYLOR ${ }^{2}$
}

\begin{abstract}
Several examples are constructed of connected Lie groups with completely reducible regular representation. An example is given in each of the following classes: (i) solvable, (ii) amenable, nonsolvable, (iii) nonamenable and (iv) non-Type I. It is also shown by example that $G$ having a completely reducible regular representation does not imply that $A(G)=B_{0}(G)$ while the reverse implication is known for separable groups (see [2] and [6).
\end{abstract}

1. Introduction. This note deals with the class of locally compact groups, $G$, with completely reducible (right) regular representation, $R^{G}$. That is, $R^{G}$ is unitarily equivalent to $\Sigma \oplus \pi_{\alpha}$, where each $\pi_{\alpha}$ is an irreducible unitary representation of $G$. The main purpose is to show by example that this class is very diverse in other properties commonly studied. Such examples could prove to be useful since some aspects of harmonic analysis simplify on such groups. This is illustrated by Khalil's work on the " $a x+b$ "-group (example (i) below) in [7].

For a locally compact group $G$, let $A(G)$ and $B(G)$ be the Fourier and Fourier-Stieltjes (resp.) algebras of $G$ (see [5] for definitions and properties). Let $B_{0}(G)=B(G) \cap C_{0}(G)$. The inclusion, $A(G) \subseteq B_{0}(G)$, always holds and usually is a strict containment as shown by the following theorem, which was proven for separable unimodular groups by Figà-Talamanca, [6], and for general separable groups by the authors, [2].

1.1 Let $G$ be a separable locally compact group. If $B_{0}(G)=A(G)$, then $R^{G}$ is completely reducible.

There has been some, unpublished, speculation that the converse of 1.1 is also true. In $\$ 3$ a counterexample is presented based on example (iii) of $\$ 2$. An element of $B_{0}\left(G_{3}\right)$ which is not in $A\left(G_{3}\right)$ is constructed, where $G_{3}$ refers to example (iii), a group with completely reducible regular representation. It may be that the converse of 1.1 holds for a restricted class of groups, such as unimodular groups.

The examples are presented in \$2. They are all connected, nonunimodular Lie groups. Several examples of totally disconnected unimodular groups with completely reducible regular representation are known. There is the group of Fell (see [1]), the construction of which has been generalized in various ways

Received by the editors September 8, 1977.

AMS (MOS) subject classifications (1970). Primary 22D10; Secondary 22D15, $43 \mathrm{~A} 35$.

'Partially supported by NSF-28697.

${ }^{2}$ National Research Council of Canada Postdoctoral Fellow. 
by Mauceri and Picardello [9]. These totally disconnected examples, together with the " $a x+b$ "-group, motivated the present authors to search for additional connected examples. A non-Type I group with completely reducible regular representation, actually a whole family of such, is constructed by Blackadar in [4]. Again, these groups are totally disconnected while example (iv) below is connected.

All of the examples of $\$ 2$ are semidirect product groups on which the Mackey induction procedure is easily applied. For the convenience of the reader a general framework that includes all of the examples is described below.

Let $G=N @ K$ be the semidirect product of a vector group $N$ and connected locally compact group $K$, where $\alpha$ is the homomorphism of $K$ into the group of automorphism of $N$. Let $\hat{N}$ denote the dual group of $N$, it is also a vector group of the same dimension as $N$. Let $\tilde{\alpha}$ denote the action of $K$ on $\hat{N}$,

$$
(\tilde{\alpha}(k) \gamma)(n)=\gamma(\alpha(k) n), \quad \text { for } \gamma \in \hat{N}, n \in N \text { and } k \in K \text {. }
$$

For each $g \in G$, let $g$ act on $\hat{N}$ via, $\gamma \rightarrow \gamma \cdot g$ where

$$
\gamma \cdot g(n)=\gamma\left(g n g^{-1}\right), \text { for } n \in N .
$$

Then $(n, k) \in G$ satisfies,

$$
\gamma \cdot(n, k)=\tilde{\alpha}(k) \gamma, \quad \text { for all } \gamma \in \hat{N} .
$$

For each $\gamma \in \hat{N}$, let $K_{\gamma}=\{k \in K: \tilde{\alpha}(k) \gamma=\gamma\}$. This is the stability group of $\gamma$ for the action $\tilde{\alpha}$ and it is a closed subgroup of $K$. Identify $N$ and $K$ with their corresponding subgroups of $G$ and let

$$
H_{\gamma}=N K_{\gamma}=\{g \in G: \gamma \cdot g=\gamma\} \text {. }
$$

The formula for an induced representation that is used below is given in [2] and it follows directly from this formula that

$$
\operatorname{ind}_{N^{\prime}}^{H_{\gamma}}(\gamma)=\gamma^{*} \otimes\left(R^{K_{r}} \circ q\right),
$$

where $\gamma^{*}$ is the extension of $\gamma$ to $H_{\gamma}$ defined by $\gamma^{*}(n k)=\gamma(n)$ and $q$ is the quotient map of $H_{\gamma}$ onto $K_{\gamma}=H_{\gamma} / N$. The regular representation of $K_{\gamma}$ is denoted $R^{K}$.

1.2 The map $\phi \rightarrow \operatorname{ind}_{H_{\gamma}}^{G}\left(\gamma^{*} \otimes(\phi \circ q)\right)$ is a one-to-one map of $\hat{K}_{\gamma}$ into $\hat{G}$. (We abuse notation by using the same symbol for an irreducible representation and its equivalence class.)

1.3 If $\gamma_{1}$ and $\gamma_{2}$ lie on the same orbit in $\hat{N}$ (that is, there is a $g \in G$ with $\left.\gamma_{1} \cdot g=\gamma_{2}\right)$, then $\operatorname{ind}_{N}^{G}\left(\gamma_{1}\right)$ is unitary equivalent to $\operatorname{ind}_{N}^{G}\left(\gamma_{2}\right)$.

$$
\begin{aligned}
R^{G} & =\operatorname{ind}_{N}^{G} R^{N}=\operatorname{ind}_{N}^{G}\left(\int_{\hat{N}} \oplus \gamma d \mu(\gamma)\right) \\
& =\int_{\hat{N}} \oplus\left(\operatorname{ind}_{N}^{G}(\gamma)\right) d \mu(\gamma),
\end{aligned}
$$


where $\mu$ is Lebesgue measure on $\hat{N}$ and equality really means unitary equivalence.

Each of the examples in $\$ 2$ has the following two properties.

(a) If $Q=\{\theta: \theta$ is an orbit in $\hat{N}$ and $\mu(\theta)>0\}$, then $\mu\left(\hat{N}-\cup_{\theta \in Q} \theta\right)=$ 0 . That is, except for a $G$-invariant null set, all the orbits in $\hat{N}$ have positive measure.

If property (a) holds then the orbits in $Q$ must be countable so write $Q=\left\{\theta_{1}, \theta_{2}, \ldots,\right\}$. For each $i$, fix a $\gamma_{i} \in \theta_{i}$ and let $K_{i}=K_{\gamma_{i}}$.

(b) For each $i$, the regular representation of $K_{i}$ is completely reducible. Write $R^{K_{i}}=\Sigma_{j} \bigoplus \phi_{j}^{i}$, for $\left\{\phi_{j}^{i}\right\} \subseteq \hat{K}_{i}$.

When $G$ has properties (a) and (b) and with $H_{i}=N K_{i}$ and $q_{i}$ the quotient map of $H_{i}$ onto $K_{i}$ one has

$$
\begin{aligned}
& R^{G}=\int_{\hat{N}} \bigoplus\left(\operatorname{ind}_{N}^{G}(\gamma)\right) d \mu(\gamma) \\
& =\sum_{i} \bigoplus \int_{\theta_{i}} \bigoplus\left(\operatorname{ind}_{N}^{G}(\gamma)\right) d \mu(\gamma) \\
& =\sum_{i} \bigoplus x_{0}\left(\operatorname{ind}_{N}^{G}\left(\gamma_{i}\right)\right) \\
& =\aleph_{0}\left(\sum_{i} \bigoplus\left(\operatorname{ind}_{H_{i}}^{G}\left(\operatorname{ind}_{N}^{H_{i}}\left(\gamma_{i}\right)\right)\right)\right) \\
& =\aleph_{0}\left(\sum_{i} \bigoplus\left(\operatorname{ind}_{H_{i}}^{G}\left(\gamma_{i}^{*} \otimes\left(R^{K_{i}} \circ q_{i}\right)\right)\right)\right) \\
& =\aleph_{0}\left(\sum_{i} \oplus\left(\sum_{j} \oplus\left(\operatorname{ind}_{H_{i}}^{G}\left(\gamma_{i}^{*} \otimes\left(\phi_{j}^{i} \circ q_{i}\right)\right)\right)\right)\right) \\
& =\aleph_{0}\left(\sum_{i, j} \oplus \sigma_{i, j}\right)
\end{aligned}
$$

where $\sigma_{i, j}=\operatorname{ind}_{H_{i}}^{G}\left(\gamma_{i}^{*} \otimes\left(\phi_{j}^{i} \circ q_{i}\right)\right)$ which is an irreducible representation of $G$ for each $i$ and $j$ in the appropriate index set. Thus $R^{G}$ is completely reducible if $G$ satisfies properties (a) and (b).

2. The examples. (i) This is the well-known " $a x+b$ "-group. Let $N=\mathbf{R}$ and $K=\mathbf{R}^{+}$, the multiplicative positive reals. Let $\alpha$ be given by $\alpha(a) b=a b$, for $a \in K$ and $b \in N$. Let $G_{1}=N @ K$.

There are two orbits of positive measure in $\hat{N}$, which is identified with $\mathbf{R}$; they are,

$$
\Theta_{1}=\{t: t>0\} \text { and } \theta_{2}=\{t: t<0\} .
$$

For each $t \in \mathcal{O}_{1} \cup \mathcal{\theta}_{2}, K_{t}=\{1\}$. Therefore properties (a) and (b) of the introduction are satisfied and $R^{G_{1}}$ is completely reducible. The usual notation is $\pi_{+}$denotes the irreducible representation obtained by inducing an element of $\theta_{1}$ while $\pi_{-}$is that obtained from an element of $\theta_{2}$. Then 


$$
R^{G_{1}}=\aleph_{0}\left(\pi_{+} \oplus \pi_{-}\right)
$$

It is easy to repeat the above construction in higher dimensions to produce an infinite number of solvable groups with completely reducible regular representations. The next two examples are nonsolvable. Example (ii) is amenable while (iii) is not.

(ii) Let $N=\mathbf{R}^{3}$ and $K=\mathbf{R}^{+} \times S O(3)$, with $\alpha$ defined by

$$
\alpha(a, M) \mathbf{x}=a(M \mathbf{x}), \quad \text { for }(a, M) \in K \text { and } \mathbf{x} \in N,
$$

where $M \mathbf{x}$ denotes the ordinary action of the matrix $M$ on the column vector x. Let $G_{2}=N @ K$. There is just one nontrivial orbit $\theta$ in $\hat{N}$ and for any $\gamma \in \mathcal{\theta}$, the stability group $K_{\gamma}$ is isomorphic to the circle group. Since $K_{\gamma}$ is compact, $R^{K_{r}}$ is completely reducible so (a) and (b) are satisfied. Thus, $R^{G_{2}}$ is completely reducible.

(iii) This example is the setting for the constructions of $\$ 3$.

Let $N=\mathbf{R}^{2}, K=\mathrm{GL}(2, \mathbf{R})^{+}$, the group of $2 \times 2$ real matrices with positive determinant. For $M \in K$, let $\alpha(M) \mathbf{x}=M \mathbf{x}$, for all column vectors $\mathbf{x} \in \mathbf{R}^{2}=$ $N$. Let $G_{3}=N @ K$.

Identifying $\hat{N}$ with $\mathbf{R}^{2}$ and considering $\gamma \in \hat{N}$ as a column vector as well, $\tilde{\alpha}(M) \gamma=M^{\tau} \gamma$, for $M \in K$ where $M^{\tau}$ is the transpose of $M$. Again, there is just one nontrivial orbit $\mathcal{\theta}$ in $\hat{N}$ so property (a) is satisfied. To check (b), fix $\gamma=\left(\begin{array}{l}q \\ 1\end{array}\right) \in \mathcal{O}$, then

$$
K_{\gamma}=\left\{\left(\begin{array}{ll}
a & b \\
0 & 1
\end{array}\right): a \in \mathbf{R}^{+}, b \in \mathbf{R}\right\}
$$

This is one of the common realizations of " $a x+b$ "-group and is easily seen to be isomorphic to $G_{1}$. With the notation of example (i).

$$
R^{K_{r}}=\aleph_{0}\left(\pi_{+} \oplus \pi_{-}\right)
$$

So

$$
R^{G_{3}}=\aleph_{0}\left(\sigma_{+} \oplus \sigma_{-}\right), \quad \text { where } \sigma_{ \pm}=\operatorname{ind}_{N K_{\gamma}}^{G_{3}}\left(\gamma^{*} \otimes\left(\pi_{ \pm} \circ q\right)\right)
$$

in the notation of the Introduction.

One can represent $\operatorname{SL}(2, \mathbf{R})$ as a homomorphic image of $G_{3}$; so $G_{3}$ is not amenable. However it is possible to show that $G_{3}$ is Type $I$, as are $G_{1}$ and $G_{2}$. This next example is non-Type $I$.

(iv) Let $N=\mathbf{R}^{5}$ and $K$ be the group of all matrices of the form,

$$
\left(\begin{array}{ccccc}
\cos t & \sin t & 0 & 0 & 0 \\
-\sin t & \cos t & 0 & 0 & 0 \\
0 & 0 & \cos 2 \pi t & \sin 2 \pi t & 0 \\
0 & 0 & -\sin 2 \pi t & \cos 2 \pi t & 0 \\
u_{1} & u_{2} & u_{3} & u_{4} & e^{t}
\end{array}\right)
$$

where $t, u_{1}, u_{2}, u_{3}, u_{4} \in \mathbf{R}$. For $M \in K$ and $\mathbf{x} \in N$, let $\alpha(M) \mathbf{x}=M \mathbf{x}$. Then 
$\tilde{\alpha}(M) \gamma=M^{\tau} \gamma$ for all $\gamma \in \hat{N}$. Let $G_{4}=N @ K$. This is a 10-dimensional solvable Lie group.

There are two nonnull orbits in $\hat{N}$,

$$
\theta_{1}=\left\{\gamma \in \hat{N}: \gamma_{5}>0\right\} \quad \text { and } \theta_{2}=\left\{\gamma \in \hat{N}: \gamma_{5}<0\right\} \text {. }
$$

Clearly $\hat{N}-\left(\theta_{1} \cup \theta_{2}\right)$ has Lebesgue measure zero so (a) is satisfied by $G_{4}$. For any $\gamma \in\left(\theta_{1} \cup \theta_{2}\right), K_{\gamma}$ is the trivial group so (b) is satisfied as well. Thus $R^{G_{4}}$ is completely reducible. In fact,

$$
R^{G_{4}}=\aleph_{0}\left(\sigma_{1} \oplus \sigma_{2}\right)
$$

where $\sigma_{i}$ is obtained from an element of $\theta_{i}(i=1,2)$ as described in the introduction.

Let $L$ be the following closed normal subgroup of $G_{4}$ consisting of elements of the form $(n, M)$, where $n=\left(0,0,0,0, x_{5}\right)$ and

$$
M=\left(\begin{array}{ccccc}
1 & 0 & 0 & 0 & 0 \\
0 & 1 & 0 & 0 & 0 \\
0 & 0 & 1 & 0 & 0 \\
0 & 0 & 0 & 1 & 0 \\
u_{1} & u_{2} & u_{3} & u_{4} & 1
\end{array}\right)
$$

$L$ is isomorphic to $\mathbf{R}^{5}$ and $G_{4} / L$ is isomorphic to the so called Mautner group which is non-Type I. Thus $G_{4}$ is not Type $\mathrm{I}$.

3. $A\left(G_{3}\right) \neq B_{0}\left(G_{3}\right)$. In this section an element of $B_{0}\left(G_{3}\right)$ is constructed which is not in $A\left(G_{3}\right)$. Thus the converse of 1.1 is false even for connected groups. Recall the notation of example (iii).

$$
\begin{aligned}
N & =\mathbf{R}^{2}, \quad K=\mathrm{GL}(2, \mathbf{R})^{+}, \quad G_{3}=N @ K, \\
\gamma & =\left(\begin{array}{l}
0 \\
1
\end{array}\right) \in \hat{N}, \quad \text { that is, } \gamma(\mathbf{x})=e^{i x_{2}}, \\
K_{\gamma} & =\left\{\left(\begin{array}{ll}
a & b \\
0 & 1
\end{array}\right): a \in \mathbf{R}^{+}, b \in \mathbf{R}\right\} .
\end{aligned}
$$

Let $H=N K_{\gamma}$ (where convenient $N$ and $K$ will be identified with the corresponding subgroup of $G_{3}$ ). Then $H=\left\{g \in G_{3}: \gamma \cdot g=\gamma\right\}$. Letting 1 stand for the trivial representation of $K_{\gamma}$ put $\chi=\gamma^{*} \otimes(1 \circ q)$, where $q$ is the quotient map of $H$ into $K_{\gamma}$. (Any element of $\hat{K}_{\gamma}$ except for $\pi_{+}$or $\pi_{-}$could be used instead of 1.) Then, $U^{x}=\operatorname{ind}_{H}^{G_{3}}(x)$ is an element of $\hat{G}_{3}$ and $U^{x}$ is disjoint from $R^{G_{3}}$ by 1.2 .

It is necessary to have an explicit formula for $U^{x}$. Of the several equivalent ways in defining induced representations we are using the formula presented in [2]. Then $U^{x}$ is a representation on $L^{2}(\theta, \mu)$, where $\theta=\mathbf{R}^{2}-\{0\}$, the orbit of $\gamma$ in $\hat{N}$ and $\mu$ is Haar measure on $\mathbf{R}^{2}$. Let $p: \theta \rightarrow G_{3}$ be a regular Borel cross-section. That is, $p(\gamma \cdot g) \in H g$ for each $g \in G_{3}, p$ is a Borel mapping, and for each compact set $A \subseteq \mathcal{O}, p(A)$ is precompact in $G_{3}$. See [8, Lemma 1.1]. 
For $g=(\mathbf{x}, M) \in G_{3}$, let $d(g)=\operatorname{det}(M)$. Then, for $f \in L^{2}(\theta, \mu), g \in G_{3}$ and $\lambda \in \mathcal{O}$,

$$
U_{g}^{\chi} f(\boldsymbol{\lambda})=d(g)^{1 / 2} f(\boldsymbol{\lambda} \cdot g) \chi_{\left\{p(\boldsymbol{\lambda}) g[p(\boldsymbol{\lambda} \cdot g)]^{-1}\right\}} .
$$

Let us fix $f$ as the characteristic function of the cube $C=(0,1] \times(0,1]$. Let $\psi(g)=\left(U_{q}^{x} f \mid f\right)$, for all $g \in G_{3}$. Then $\psi \in B\left(G_{3}\right)$ by definition of $B\left(G_{3}\right)$ but $\psi$ is not element of $A\left(G_{3}\right)$. For, if $\psi$ were in $A\left(G_{3}\right)$, then there would be a $\eta \in L^{2}\left(G_{3}\right)$ with

$$
\psi(g)=\left(R_{g}^{G_{3}} \eta \mid \eta\right), \text { for all } g \in G_{3} .
$$

Then, $U^{x}$ would be unitarily equivalent to the restriction of $R^{G_{3}}$ to the cyclic subspace of $L^{2}\left(G_{3}\right)$ spanned by $\eta$, which is not true.

It will be shown that $\psi$ vanishes at infinity; hence, $\psi \in B_{0}\left(G_{3}\right)-A\left(G_{3}\right)$ as desired.

Let $\phi(M)=\operatorname{det}(M)^{1 / 2} \mu\left[\left(M^{\tau}\right)^{-1} C \cap C\right]$, for $M \in G L(2, \mathbf{R})^{+}$. The following lemma is essential in showing that $\psi$ vanishes at infinity.

3.1 Lemma. $\phi$ vanishes at infinity on $\mathrm{GL}(2, \mathbf{R})^{+}$.

Proof. Let $\varepsilon>0$ be given. Then

$$
|\phi(M)| \leqslant \min \left\{\operatorname{det}(M)^{1 / 2}, \operatorname{det}(M)^{-1 / 2}\right\}<\varepsilon,
$$

if $\operatorname{det}(M)$ is outside of $\left[\varepsilon^{2}, 1 / \varepsilon^{2}\right]$. Suppose $\operatorname{det}(M)$ lies in this compact interval. Let $M_{0}=\left(\operatorname{det}(M)^{-1 / 2} M\right)$, then $M_{0} \in \operatorname{SL}(2, \mathbf{R})$. Write $\left(M_{0}^{\tau}\right)^{-1}=$ $R U$, where $R$ is a rotation matrix and $U$ is of the form $\left(\begin{array}{ll}a & b \\ 0 & 1 / a\end{array}\right)$, for $a \in \mathbf{R}^{+}$and $b \in \mathbf{R}$. Then $\left(M^{\tau}\right)^{-1}$ is the rotation by $R$ of the parallelogram, $P$, determined by

$$
\operatorname{det}(M)^{-1 / 2}\left(\begin{array}{c}
b \\
1 / a
\end{array}\right) \text { and } \operatorname{det}(M)^{-1 / 2}\left(\begin{array}{l}
a \\
0
\end{array}\right) .
$$

Clearly $\mu\left(\left(M^{\tau}\right)^{-1} C \cap C\right) \leqslant \mu(P \cap C)$. Since $\operatorname{det}(M)^{-1 / 2} \leqslant 1 / \varepsilon$ a simple geometric argument shows that there is a compact subset, $D$, of $\mathbf{R}^{+} \times \mathbf{R}$ such that $\mu(P \cap C)<\varepsilon^{2}$ whenever $M \in \mathrm{GL}(2, \mathbf{R})^{+}$is such that $\operatorname{det}(M) \in$ $\left[\varepsilon^{2}, 1 / \varepsilon^{2}\right]$ and $(a, b)$ is outside $D$. Let $\Omega$ be the set of all matrices of the form $\left((d R U)^{\tau}\right)^{-1}$, where $d \in\left[\varepsilon^{2}, 1 / \varepsilon^{2}\right], R$ is a rotation matrix and $U=\left(\begin{array}{cc}a & b \\ 0 & 1 / a\end{array}\right)$ for $(a, b) \in D$. Then $\Omega$ is a compact subset of $\mathrm{GL}(2, \mathbf{R})^{+}$and $|\phi(M)|<\varepsilon$, if $M \in \mathrm{GL}(2, \mathbf{R})^{+}-\Omega$.

3.2 Proposition. $\psi \in B_{0}\left(G_{3}\right)-A\left(G_{3}\right)$.

Proof. It is necessary to show that $\psi$ vanishes at infinity. Let $\varepsilon>0$ be given. Write a general element $g$ of $G_{3}$ as $(\mathbf{x}, M)$ for $\mathbf{x} \in \mathbf{R}^{2}$ and $M \in$ GL $(2, \mathbf{R})^{+}$. Then 


$$
\begin{aligned}
|\psi(g)| & =\left|\int_{\theta} U_{g}^{\chi} f(\lambda) f(\lambda) d \lambda\right| \\
& =\left|\int_{\theta} d(g)^{1 / 2} f(\lambda \cdot g) \bar{f}(\lambda) \chi\left(p(\lambda) g[p(\lambda \cdot g)]^{-1}\right) d \lambda\right| \\
& \leqslant \int_{\theta} d(g)^{1 / 2}|f(\lambda \cdot g) \bar{f}(\lambda)| d \lambda \\
& =\operatorname{det}(M)^{1 / 2} \mu\left[C \cdot g^{-1} \cap C\right] \\
& =\operatorname{det}(M)^{1 / 2} \mu\left[\left(M^{\tau}\right)^{-1} C \cap C\right]=\phi(M) .
\end{aligned}
$$

By 3.1, there is a compact set $\Omega \subseteq \mathrm{GL}(2, \mathbf{R})^{+}$, such that $|\psi(g)|<\varepsilon$ if $g=(\mathbf{x}, M)$ and $M \in \mathrm{GL}(2, \mathbf{R})^{+}-\Omega$. To deal with the variable $\mathbf{x}$, note that $\gamma \cdot p(\lambda)=\lambda$, by definition of $p$, and

$$
\begin{aligned}
\chi\left(p(\lambda) g[p(\lambda \cdot g)]^{-1}\right) & =(\gamma \cdot p(\lambda))(\mathbf{x}) \chi\left(p(\lambda)(0, M)[p(\lambda \cdot g)]^{-1}\right) \\
& =\chi\left(p(\lambda)(\mathbf{x}, I) p(\lambda)^{-1}\right)\left(p(\lambda)(0, M)[p(\lambda \cdot g)]^{-1}\right) \\
& =\lambda(\mathbf{x}) \chi\left(p(\lambda)(0, M)[p(\lambda \cdot g)]^{-1}\right) .
\end{aligned}
$$

Fix $M \in \Omega$ and let

$$
F_{M}(\lambda)=\operatorname{det}(M)^{1 / 2} f(\lambda \cdot g) \bar{f}(\lambda) \chi\left(p(\lambda)(0, M)[p(\lambda \cdot g)]^{-1}\right) .
$$

Note that $\lambda \cdot g=M^{\tau} \lambda$ so $F_{M}$ is independent of $\mathbf{x}$. Considering $F_{M}$ as an element of $L^{1}\left(\mathbf{R}^{2}\right)$,

$$
|\psi(\mathbf{x}, M)|=\left|\hat{F}_{M}(\mathbf{x})\right|=\left|\int F_{M}(\lambda) \lambda(\mathbf{x}) d \lambda\right| .
$$

The Fourier transform, $\hat{F}_{M}$ vanishes at infinity by the usual Riemann-Lebesgue lemma. Hence, there is a compact set $A_{M}$ in $\mathbf{R}^{2}$ such that

$$
|\psi(\mathbf{x}, M)|<\varepsilon / 2, \quad \text { if } \mathbf{x} \in \mathbf{R}^{2}-A_{M} .
$$

The map $M \rightarrow F_{M}$ is clearly continuous from $\operatorname{GL}(2, \mathbf{R})^{+}$into $L^{1}\left(\mathbf{R}^{2}\right)$ and $F_{M} \rightarrow \hat{F}_{M}$ is continuous from $L^{1}\left(\mathbf{R}^{2}\right)$ into $C_{0}\left(\mathbf{R}^{2}\right)$. Therefore, there is a uniform compact set $A \subseteq \mathbf{R}^{2}$ such that

$$
|\psi(\mathbf{x}, M)|<\varepsilon, \quad \text { if } g=(\mathbf{x}, M) \in G_{3}-(A \times \Omega) .
$$

Thus $\psi$ vanishes at infinity.

\section{REFERENCES}

1. L. Baggett, A separable group having a discrete dual is compact, J. Functional Anal. 10 (1972), 131-148.

2. L. Baggett and K. Taylor, A sufficient condition for complete reducibility of the regular representation (to appear).

3. , Riemann-Lebesgue subsets of $\mathbf{R}^{n}$ and representations which vanish at infinity, $\mathbf{J}$. Functional Anal. (to appear).

4. B. Blackadar, The regular representation of restricted direct product groups, J. Functional Anal. 25 (1977), 267-274.

5. P. Eymard, L'algèbre de Fourier d'un groupe localement compact, Bull. Soc. Math. France 92 (1964), 181-236. 
6. A. Figà-Talamanca, Positive definite functions which vanish at infinity, Pacific J. Math. 69 (1977), 355-363.

7. I. Khalil, Sur l'analyse harmonique du groupe affine de la droite, Studia Math. 51 (1974), 139-167.

8. G. Mackey, Induced representations of locally compact groups. I, Ann. of Math. (2) 55 (1952), 101-139.

9. G. Mauceri and M. Picardello, Noncompact unimodular groups with purely atomic Plancerel measures (to appear).

Department of Mathematics, University of Colorado, Boulder, Colorado 80309

Department of Mathematics, University of Saskatchewan, Saskatoon, Saskatchewan, CANADA S7N OWO 